\title{
REFERENCES
}

The full list of references and further reading is published with the online version of this article at www.australianprescriber.com/magazine/37/6/192/5

8. Austin PF, Bauer SB, Bower W, Chase J, Franco I, Hoebeke P, et al. The standardization of terminology of lower urinary tract function in children and adolescents: update report from the Standardization Committee of the International Children's Continence Society. J Urol 2014;191:1863-5.
9. National Institute for Health and Care Excellence. Nocturnal enuresis: the management of bedwetting in children and young people. NICE Guidelines [CG111]. 2010 www.nice.org.uk/guidance/cg111 [cited 2014 Nov 3]

\section{Top 10 drugs}

\section{Table 1 Top 10 drugs by DDD/1000 pop/day *+}

\begin{tabular}{llr}
\hline Constituent drug & PBS/RPBS $\ddagger$ \\
\hline 1. & atorvastatin & 69.31 \\
2. & rosuvastatin & 41.87 \\
3. & paracetamol & 33.65 \\
4. & perindopril & 32.73 \\
5. & irbesartan & 28.49 \\
6. & amlodipine & 26.73 \\
7. & esomeprazole & 24.90 \\
8. & candesartan & 23.38 \\
9. & ramipril & 22.43 \\
10. & simvastatin & 17.94 \\
\hline
\end{tabular}

\section{Table 2 Top 10 drugs by prescription counts ${ }^{\dagger}$}

\begin{tabular}{llc}
\hline Drug & PBS/RPBS $\ddagger$ \\
\hline 1. & atorvastatin & 8659127 \\
2. rosuvastatin & 7610977 \\
3. esomeprazole & 6967801 \\
4. paracetamol & 6299698 \\
5. pantoprazole & 4179926 \\
6. perindopril & 3951282 \\
7. metformin hydrochloride & 3539513 \\
8. salmeterol and fluticasone & 3168825 \\
9. irbesartan & 3032109 \\
10. simvastatin & 2974311 \\
\hline
\end{tabular}

\section{Table 3 Top 10 drugs by cost to government ${ }^{+}$}

\begin{tabular}{|c|c|c|c|c|}
\hline \multicolumn{2}{|c|}{ Drug } & \multirow{2}{*}{$\begin{array}{r}\text { Cost to government } \\
\text { (A\$) }\end{array}$} & \multirow{2}{*}{$\begin{array}{r}\begin{array}{r}\text { DDD/1000 pop/day * } \\
\text { PBS/RPBS } \ddagger\end{array} \\
41.87\end{array}$} & \multirow{2}{*}{$\begin{array}{c}\begin{array}{c}\text { Prescriptions } \\
\text { PBS/RPBS }\end{array} \\
7610977\end{array}$} \\
\hline 1. & rosuvastatin & & & \\
\hline 2. & adalimumab & 270237753 & 0.50 & 152563 \\
\hline 3. & atorvastatin & 259067466 & 69.31 & 8659127 \\
\hline 4. & esomeprazole & 204253775 & 24.90 & 6967801 \\
\hline 5. & etanercept & 153005371 & 0.29 & 87283 \\
\hline 6. & insulin glargine & 131811872 & 6.94 & 320806 \\
\hline 7. & tiotropium bromide & 131776620 & 7.03 & 1993769 \\
\hline 8. & fingolimod & 113531100 & 0.16 & 49576 \\
\hline 9. & olanzapine & 109682018 & 3.09 & 993826 \\
\hline & quetiapine & 99773937 & 2.62 & 978353 \\
\hline
\end{tabular}

* The defined daily dose (DDD)/thousand population/day is a more useful measure of drug utilisation than prescription counts. It shows how many people in every thousand Australians are taking the standard dose of a drug every day. DDD includes use in combination products.

+ Based on date of supply. Does not include private prescriptions or prescriptions under PBS co-payment.

‡ PBS Pharmaceutical Benefits Scheme, RPBS Repatriation Pharmaceutical Benefits Scheme

Source: Department of Health, 10 October 2014. (c) Commonwealth of Australia.

The cost figures exclude any patient contributions. 